Developing an emergency department crowding dashboard: a design science approach

Peer-reviewed author version

MARTIN, Niels; BERGS, Jochen; Eerdekens, Dorien; DEPAIRE, Benoit \& Verelst, Sandra (2018) Developing an emergency department crowding dashboard: a design science approach. In: International Emergency Nursing, 39, p. 68-76.

DOI: 10.1016/j.ienj.2017.08.001

Handle: http://hdl.handle.net/1942/25633 


\section{Developing an emergency department crowding dashboard: a design science approach}

Niels Martin ${ }^{1}$, Jochen Bergs ${ }^{1}$, Dorien Eerdekens ${ }^{1}$, Benoît Depaire ${ }^{1}$, Sandra Verelst ${ }^{2}$

\section{ABSTRACT}

Background: As an emergency department (ED) is a complex adaptive system, the analysis of continuously gathered data is valuable to gain insight in the real-time patient flow. To support the analysis and management of ED operations, relevant data should be provided in an intuitive way.

Aim: Within this context, this paper outlines the development of a dashboard which provides real-time information regarding ED crowding.

Methods: The research project underlying this paper follows the principles of design science research, which involves the development and study of artifacts which aim to solve a generic problem. To determine the crowding indicators that are desired in the dashboard, a modified Delphi study is used. The dashboard is implemented using the open source Shinydashboard package in $\mathrm{R}$.

Results: A dashboard is developed containing the desired crowding indicators, together with general patient flow characteristics. It is demonstrated using a dataset of a Flemish ED and fulfills the requirements which are defined a priori.

Conclusions: The developed dashboard provides real-time information on ED crowding. This information enables ED staff to judge whether corrective actions are required in an effort to avoid the adverse effects of ED crowding.

KEYWORDS: emergency department; crowding; dashboard; data analysis; ED management; design science research

\footnotetext{
${ }^{1}$ Hasselt University, Belgium

2 Leuven University Hospitals, Belgium
} 
This is an Accepted Manuscript of an article published by Elsevier in International Emergency Nursing, available online: https://doi.org/10.1016/j.ienj.2017.08.001

\section{INTRODUCTION}

\section{Research problem}

The fact that most emergency departments (EDs) are often crowded is well known amongst healthcare professionals and repeatedly described in literature (Boyle et al., 2012; Pines et al., 2011; Pitts et al., 2012; Verelst et al., 2014). Emergency department (ED) crowding has been linked to negative consequences for both patients (e.g., mortality and morbidity) and caregivers (e.g., work related stress and burnout) (Johnson and Winkelman, 2011).

The current research paradigm is concerned with predicting and controlling - predicting when crowding happens and controlling the causal factors to prevent crowding from happening. To predict the occurrence of crowding, researchers have tried to single out the most important causal factors - often positioned within Asplin's model (Asplin et al., 2003), followed by intervention studies seeking to control aspects of ED operations in order to prevent crowding from happening. However, the context in which ED crowding takes place is characterised by a complex adaptive system (CAD) (Chinnis and White, 1999). The ED is a complex macroscopic collection of partially connected micro-structures (agents), formed in order to adapt to the changing environment, such that its survivability as a macro-structure increases. The system is complex in the sense that it is a dynamic network of interactions, in which the relationships compel more than aggregations of individual static entities. The system is adaptive in the sense that the individual and collective behaviour mutates and organises itself according to the change-initiating micro-event or collection of events. Microscopic impulses at the level of the individual agent can generate macroscopic effects. The adaptive character of the system can be powerful if it is sufficiently sensitive to anticipate upon changes in the environment. However, the peculiar structure of critical networks can allow macroscopic "avalanches" to take place. In the context of ED crowding this means full waiting rooms and unsafe conditions.

As the behaviour of such a complex adaptive system is hard to predict and control, it has been suggested to alter the research paradigm towards analysing and managing (Bergs et al., 2016). Analysing refers to the use of continuously gathered data. The introduction of information and communication technology (ICT) tools, electronic patient tracking, electronic 
This is an Accepted Manuscript of an article published by Elsevier in International Emergency Nursing, available online: https://doi.org/10.1016/j.ienj.2017.08.001

patient files and so on, has provided us a mass of data related to occupancy, waiting time and other relevant operational measures and indicators (Ryu and Song, 2014). These data should be analysed in order to create questions and answers that really matter and to gain insight into what is happening without relying on proxy measures, predicted outcomes, or gut feeling (Kamal, 2014). It is important to realise that these data are the reflection of a process, which is executed for each patient. Gaining useful insights requires analytical techniques that consider and exploit the process dimension within the data. Managing refers to the proactive management of the system in order to prevent devastating "avalanches". Instead of waiting until the ED is crowded and patients need to be placed in the hallway, changes in indicators must be recognised and managed accordingly to prevent situations of poor quality and safety. This requires a systems approach, which, besides changes in the basic structure of the organisation, also comprises continuous analysis, ad hoc adaptations of procedures, and adjustments to reduce the effect of causal factors.

\section{Study purpose}

The rationale behind this study is situated in the aforementioned need for real-time information regarding ED crowding. More specifically, the purpose of the study is the development of a dashboard designed to provide this information. This will increase the sensitivity of the ED as an adaptive system and enable staff members to proactively judge whether corrective actions are required. By supporting ED analysis and management, it is an asset towards achieving higher levels of quality and safety.

\section{Research question}

As a first step in a project aimed to improve quality and safety in an ED, a system was needed that delivers real-time information regarding ED crowding. To this end, it needs to be investigated how a system fulfilling this need should be developed. This paper describes the development of such a system, which should present the information deemed desirable by ED staff in an intuitive way.

\section{METHODS}

From a methodological perspective, this study follows the principles of design science research (DSR). DSR involves the development and study of artifacts, which are human- 
This is an Accepted Manuscript of an article published by Elsevier in International Emergency Nursing, available online: https://doi.org/10.1016/j.ienj.2017.08.001

made objects that aim to solve a generic problem experienced in practice (Johannesson and Perjons, 2014). While natural sciences tend to focus on problem understanding and explanation, the central goal of DSR is problem solving (Niehaves, 2007), which underlines its relevance for the topic of this paper. In this paper, the artifact corresponds to the dashboard, which addresses the need to have a workable process monitoring tool to gain real-time insight in ED crowding.

In order to select the crowding indicators that need to be included in the dashboard, a modified two-round Delphi study is used. The central premise of Delphi is founded upon the assumption that collective group opinion is more valid than personal opinion alone (Hasson et al. 2000). The Delphi technique was chosen as a method to develop consensus in a group who would reflect national ED management. In the first round, a questionnaire is distributed to the heads of department of all Flemish EDs. The responses are summarised and presented to experts in the second round, which explains the modified character of the Delphi study. Consultation of experts is likely to improve the quality of the received feedback for the purpose of the study.

\section{Design science research}

The research project underlying this paper follows the principles of design science research (DSR). For a detailed discussion on DSR, the reader is referred to, e.g., Johannesson and Perjons (2014), Wieringa (2014) and Dresch et al. (2015). In literature, several frameworks have been proposed which outline the key activities that need to be conducted when performing DSR. Given its clear structure, this paper uses the framework of Johannesson and Perjons (2014), which is based on the work of Peffers et al. (2007). The framework describes five DSR activities, which can be revisited at any stage of research: (i) problem explication, (ii) artifact and requirement definition, (iii) artifact design and development, (iv) artifact demonstration and $(v)$ artifact evaluation. Each of these five activities will be briefly discussed within the problem context under consideration.

Firstly, the problem at hand needs to be studied and its relevance has to be shown. From the introduction, it follows that a shift towards analysing and managing an ED as a complex 
This is an Accepted Manuscript of an article published by Elsevier in International Emergency Nursing, available online: https://doi.org/10.1016/j.ienj.2017.08.001

adaptive system requires the presence of real-time information on ED crowding. Moreover, it should be determined which crowding measures are deemed desirable by ED staff.

Secondly, the artifact that will be developed is defined, and its requirements are identified. The artifact that will be developed is a dashboard containing real-time general patient and patient flow statistics and a set of crowding indicators. To select the most desirable crowding indicators, a modified two-round Delphi technique is used to establish consensus amongst a panel of nurses and clinicians drawn from an ED background, with experience in operational managing. The Delphi technique is a well-established hybrid research method that combines both qualitative and quantitative approaches (Keeney et al. 2001). As indicated in the introduction, a questionnaire is distributed in the first round to the heads of department of all Flemish EDs (February 2016). The input obtained from 26 questionnaires is summarised and presented to 6 experts in the second round (March 2016). Based on their input, the measures that are included in the dashboard are selected. The results of the modified Delphistudy are also used to specify requirements for the artifact, which are listed in the results section.

Thirdly, the artefact is designed and developed. The dashboard is implemented using the Shinydashboard package (2016) in R (2016). R is an open source software language which provides extensive functionalities for data manipulation, data analysis and the creation of graphs. Shiny (2016) is an open source web application framework which enables users to transform their analyses into interactive web applications. The Shinydashboard package builds upon these foundations and has a special focus on dashboard development using Shiny.

Fourthly, the developed artifact is applied to a specific problem instance to demonstrate its feasibility. To this end, the results section outlines the dashboard's operations using a reallife dataset of an ED. The available dataset contains anonymised data of 4451 patients, recorded by the information system of a Belgian ED in April 2011. Besides basic personal information such as gender and age, the dataset also contains medical information such as the triage code, the ED unit to which the patient is assigned and an indication of whether the patient is discharged at the ED. Moreover, dates and times are included for: the assignment of a treatment room; the triage code assignment; the request of the first lab test; the 
This is an Accepted Manuscript of an article published by Elsevier in International Emergency Nursing, available online: https://doi.org/10.1016/j.ienj.2017.08.001

delivery of the first lab result; the delivery of the last lab result; the request of the first radiological examination; the execution of the first radiological examination; the delivery of the first radiological report; The delivery of the last radiological report; The retrieval of drugs from the electronic cabinet; the request of a hospital bed (in case of admittance to the hospital); the assignment of a hospital bed (in case of admittance to the hospital); the time at which a patient leaves the ED. The dataset also includes data on the average number of patients that is present in the ED during the $x$-th hour of presence of this patient, with $x$ ranging from 1 to 8 . This provides insight in the pressure on the ED during the stay of a particular patient.

Finally, the artifact is evaluated by determining the degree to which it meets the requirements. This was done by a multi-disciplinary team.

\section{Ethical considerations}

All participation was voluntary and participants were made aware of their right to withdraw from the study at any point without explanation. Participants were informed that the collected information would be kept confidential and that the questionnaire was anonymous. There were no incentives provided for completing the questionnaire. A full proposal outlining all study methods and stages was reviewed by the institutional review board, who granted permission for the study to proceed and waived the necessity of an informed consent given the study methodology. The act of completing each questionnaire was interpreted as consent to participate in the study.

\section{RESULTS}

\section{Requirement specification}

The dashboard's central goal is to provide a real-time overview of ED crowding. Using this information, staff members can proactively judge whether corrective actions are required. To operationalize this goal, requirements are specified. Consistent with Johannesson and Perjons (2014), a distinction is made between functional and non-functional requirements. 
This is an Accepted Manuscript of an article published by Elsevier in International Emergency Nursing, available online: https://doi.org/10.1016/j.ienj.2017.08.001

While the former specify the functions that the artifact needs to provide, the latter are related to the artifact's structure and its environment.

Based on the results of the modified Delphi study, two functional requirements for the dashboard are put forward:

- The dashboard should represent and visualize ED crowding in a non-binary way such that the user can judge the current situation.

- The dashboard has to provide real-time information.

Besides these functional requirements, four non-functional requirements are also specified:

- The dashboard needs to present its content in a clear and concise way. Even though the dashboard has to provide a complete view on the ED status, staff members should be able to analyze the provided information in a short time span.

- The dashboard should be usable in multiple EDs and, hence, has to be generic and standardized. In this way, it can be implemented in another ED with minimal adjustments.

- The dashboard has to provide information unambiguously. When visualisations are used, these should not be prone to misinterpretations.

- The information that is used and the visualisations should adhere to privacy regulations.

\section{Dashboard design and development}

\section{Dashboard use case}

Given the dashboard's goal to provide ED staff with comprehensive crowding information, Figure 1 visualises the dashboard's use case. The user interacts with the dashboard by consulting it and receiving real-time information. Based on this information, the user can decide whether corrective actions are required. When measures are taken, this will impact the dashboard as they aim to influence patient flow. The dashboard can be used to evaluate the effect of the corrective actions to determine whether further adjustments are required. 
This is an Accepted Manuscript of an article published by Elsevier in International Emergency Nursing, available online: https://doi.org/10.1016/j.ienj.2017.08.001

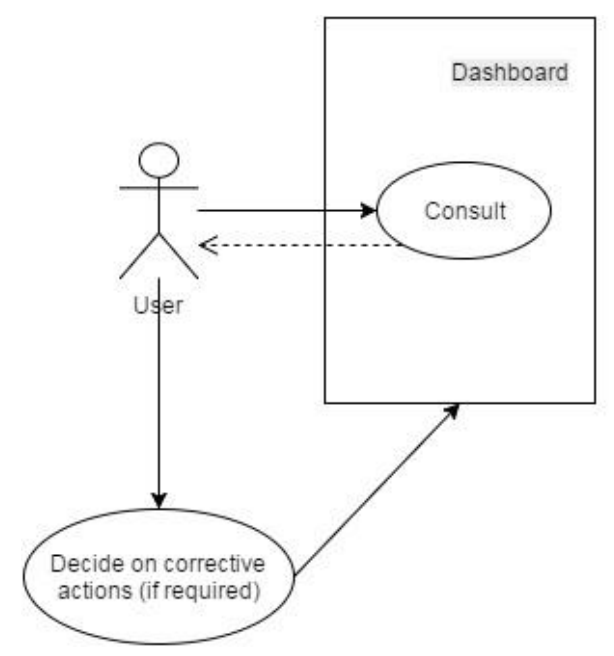

Figure 1: Use case diagram

\section{Dashboard structure}

The general structure of the dashboard is presented in Figure 2. From this diagram, it follows that the dashboard requires a limited number of settings and provides information divided in seven content tabs. In Figure 3, depicting a screenshot of the dashboard, three parts are distinguished using rectangles with rounded corners. In the top left part (red rectangle), the user has to specify some settings such as the number of nurses, the number of doctors and the number of available beds. The lower left part (green rectangle) lists the tabs that are available and from which the content is visualized at the right (blue rectangle). Using the results of the modified Delphi study as an input, the dashboard's content tabs are defined. A full overview over of the dashboard's layout is provided in Appendix. 
This is an Accepted Manuscript of an article published by Elsevier in International Emergency Nursing, available online: https://doi.org/10.1016/j.ienj.2017.08.001

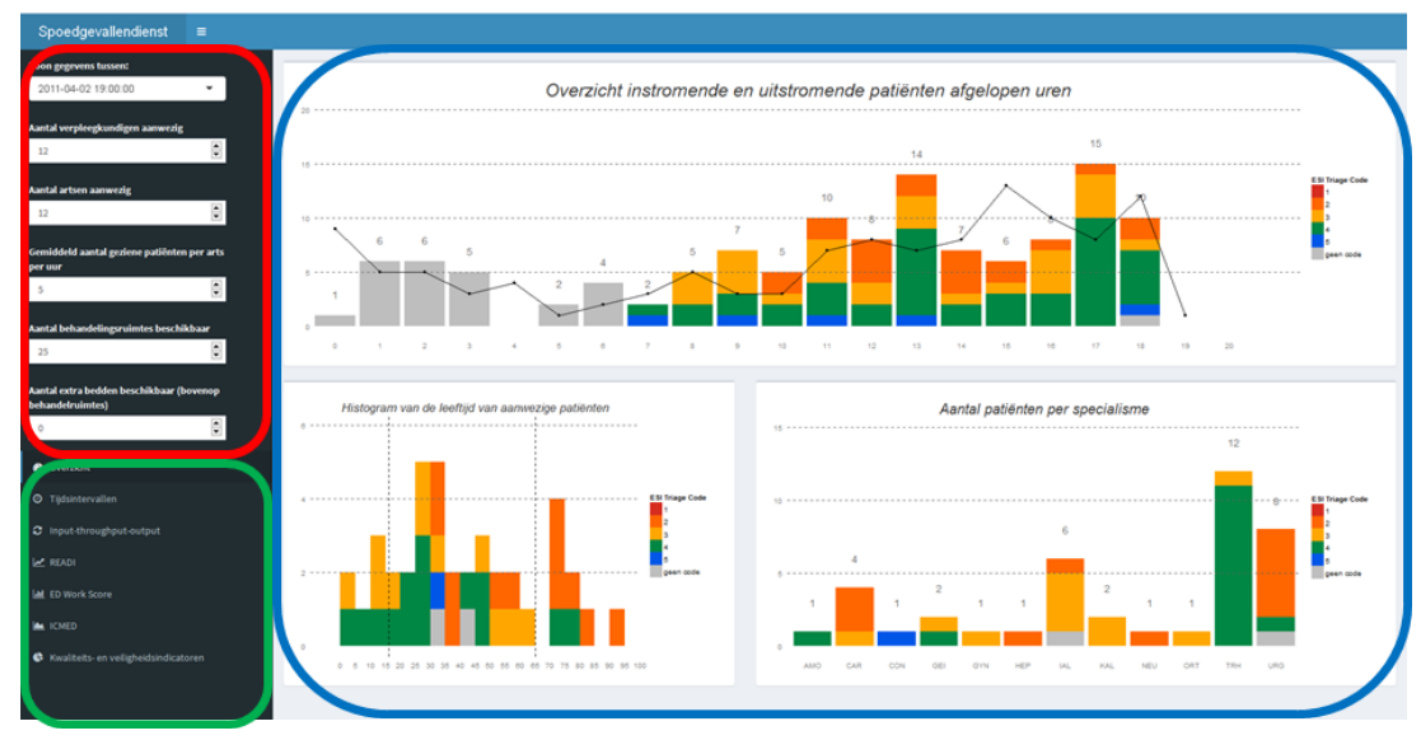

Figure 3: Screenshot of dashboard structure 
This is an Accepted Manuscript of an article published by Elsevier in International Emergency Nursing, available online: https://doi.org/10.1016/j.ienj.2017.08.001

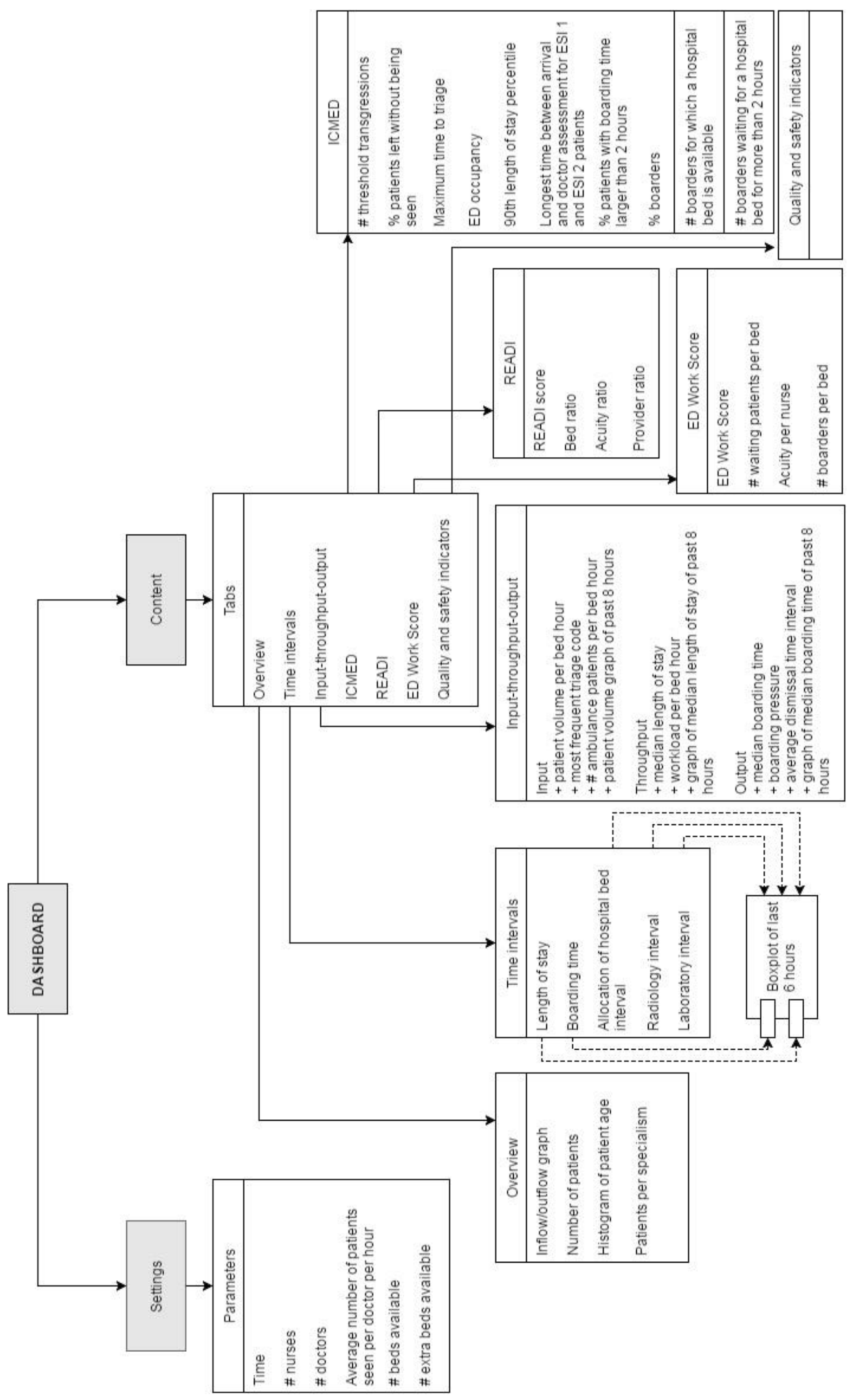

Figure 2: Diagram of dashboard structure 
This is an Accepted Manuscript of an article published by Elsevier in International Emergency Nursing, available online: https://doi.org/10.1016/j.ienj.2017.08.001

\section{Dashboard demonstration}

To simulate real-time support, the dashboard was initialised on April 1st, 2011 at 7pm. The number of nurses and doctors are set to 12 and the number of beds equals 25 . While a detailed description of all indicators is beyond the scope of this paper, this section aims to demonstrate the applicability of the dashboard in a real-life context.

Given the settings outlined above, the overview tab of the dashboard indicates that 60 patients are present at the ED. As shown in Figure 4, the overview tab highlights that arriving patients are primarily of triage codes 3 and 4 . Moreover, the age histogram indicates that mainly geriatric patients are present. The histogram at the bottom right shows that ED units with code IAL and TRH have to deal with the highest number of patients.

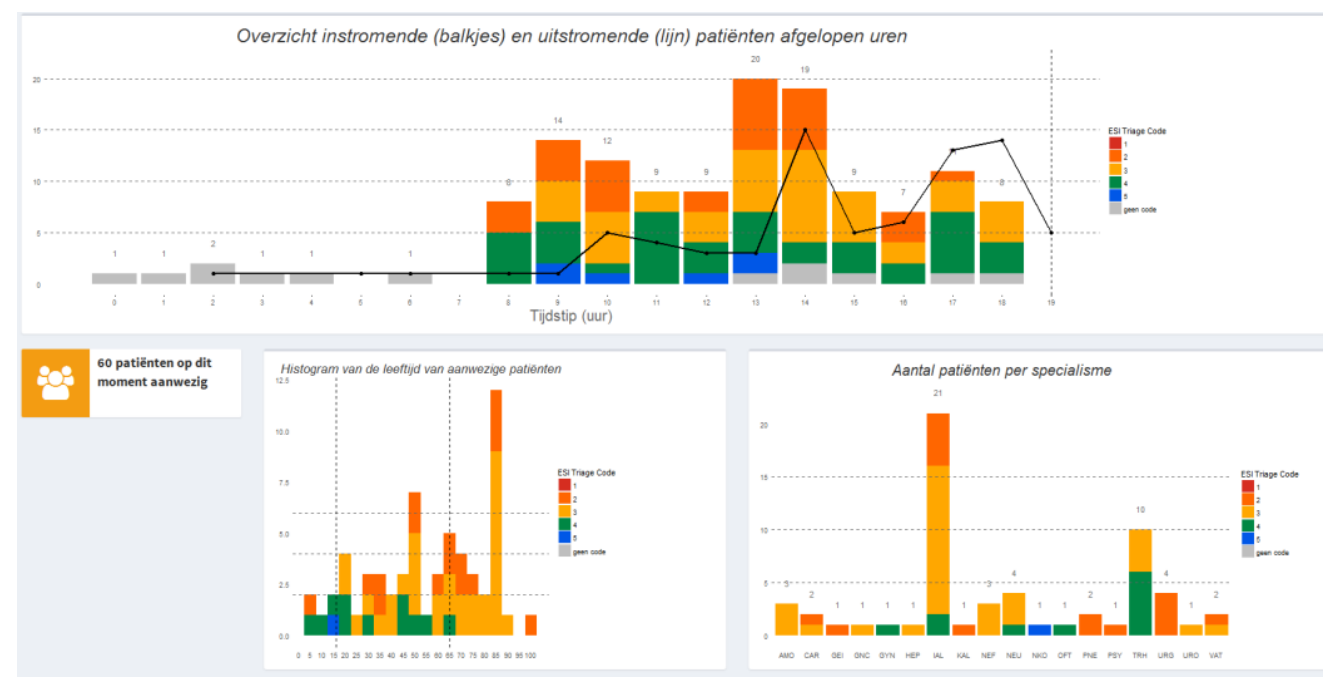

Figure 4: Screenshot of overview tab on April 1st, 2011 (7pm)

The input-throughput-output tab in Figure 5 indicates that the throughput in the ED and the transfer to the hospital are troublesome with a median length of stay of 272 minutes and 30 boarders. The fact that hospital transfers pose a problem is confirmed by the ICMED indicators, as shown in Figure $6.93 \%$ of all boarders are waiting over 2 hours to be transferred to a hospital ward. Moreover, for 18 of the 30 boarders, a hospital bed is already available. Note that the ICMED indicators indicate a crowded ED as 6 threshold 
This is an Accepted Manuscript of an article published by Elsevier in International Emergency Nursing, available online: https://doi.org/10.1016/j.ienj.2017.08.001

transgressions are observed. A similar conclusion is reached in the READI and ED Work Score tab.

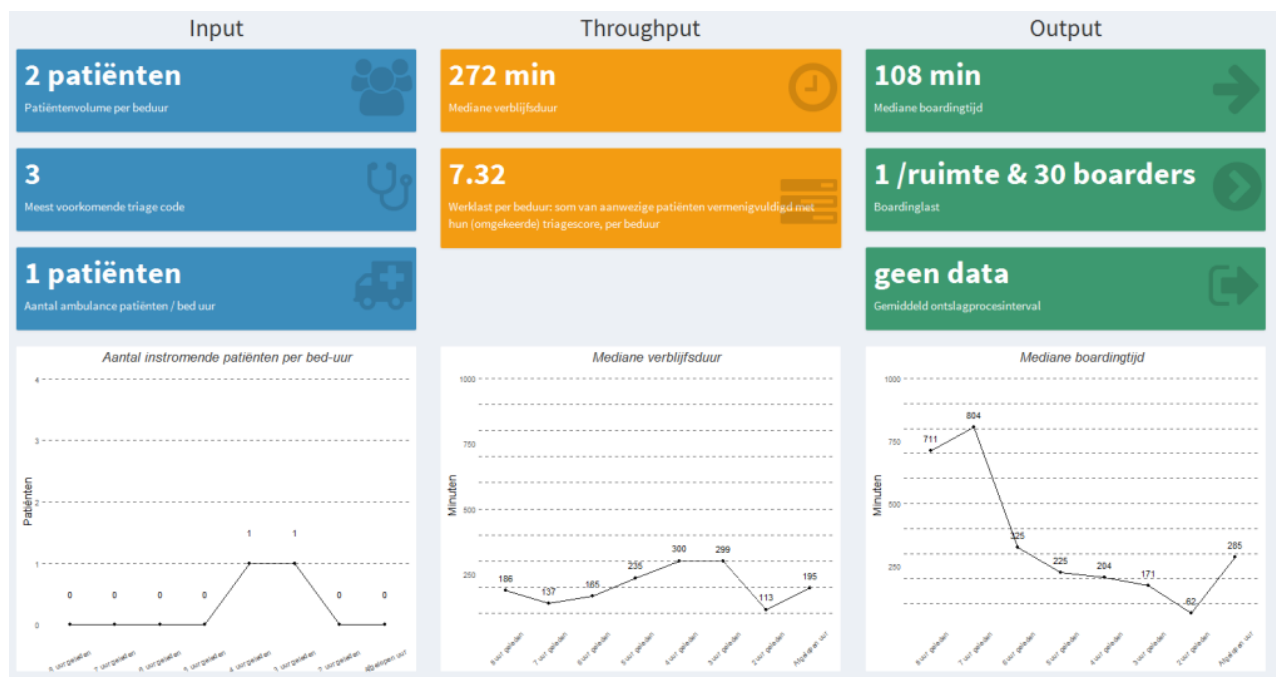

Figure 5: Screenshot of input-throughput-output tab on April, $1^{\text {st }}(7 p m)$

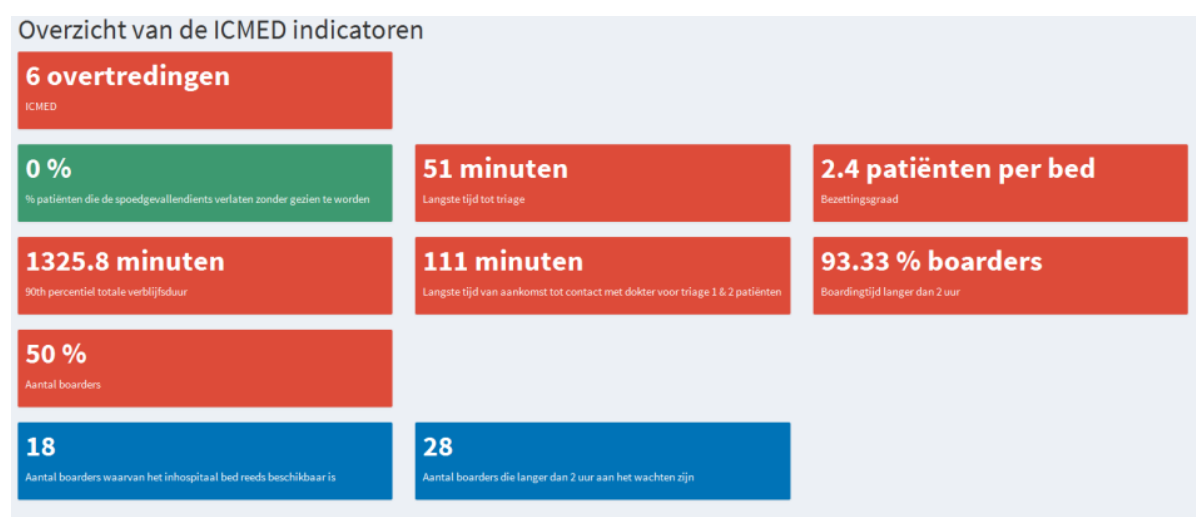

Figure 6: Screenshot of ICMED tab on April, $1^{\text {st }}(7 p m)$

\section{Dashboard evaluation}

In DSR, evaluation comes down to determining to which degree the developed artifact meets its requirements (Johannesson and Perjons, 2014). This section outlines the extent to which the presented dashboard meets the requirements formulated above.

The first requirement indicates that the degree of crowding should be represented and visualised in a non-binary way. The proposed dashboard fulfills these conditions as it includes 
This is an Accepted Manuscript of an article published by Elsevier in International Emergency Nursing, available online: https://doi.org/10.1016/j.ienj.2017.08.001

a series of crowding indicators defined in literature. Moreover, the values of these indicators are given, without a value judgment. Consequently, no binary assessment on ED crowding is made as the interpretation of the numeric values is left to the user.

The second requirement relates to the real-time character of the dashboard. Depending on the time at which the dashboard is consulted, the indicators and graphs will be adjusted to reflect the ED status at a particular point in time. This is also simulated for demonstration purposes. Consequently, the dashboard has the capacity to provide real-time support when real-time information is inserted to the data table underlying the dashboard.

Requirement three stipulates that information needs to be provided in a clear and concise way. The dashboard presents a limited number of crowding indicators, grouped in tabs. From the wide range of measures defined in literature, the most desirable ones are selected by means of a modified Delphi study. Moreover, the overview tab allows the user to quickly gain insight in the status of the ED. Therefore, it can be concluded that the dashboard presents its content in a clear and concise way.

The fourth requirement states that the dashboard should be sufficiently generic such that it can be used in multiple EDs. The dashboard's reusability is ensured by the settings that the user can specify, such as the number of nurses and the number of beds. Using these settings, the calculation basis of the crowding indicators can easily be adjusted to the specificities of an ED. Regarding the dashboard's content, it should be noted that all indicators originate from literature and the included measures are selected using the tworound modified Delphi study outlined in the methods section.

Requirement five indicates that information should be provided unambiguously. For graphical visualisations, the lie-factor of Tufte (2001) can be considered, which is calculated by dividing the effect size in the visualization by the effect size in the data. When the resulting value lies between 0.95 and 1.05 , the graph can be deemed to be free from distortions as the latter can be misleading for the user. Within the proposed dashboard, no differences are present between the effect sizes in the data and the visualisations. Consequently, the liefactor conditions are satisfied. Besides graphs, the dashboard only provides numerical values as the user is responsible for their interpretation. Consequently, all information is presented in an unambiguous way, which minimises the risk for misinterpretations. 
This is an Accepted Manuscript of an article published by Elsevier in International Emergency Nursing, available online: https://doi.org/10.1016/j.ienj.2017.08.001

The final requirements demand adherence of privacy regulations. The dashboard does not require personal information or medical details of identifiable patients, as shown in its demonstration using an anonymized dataset. Moreover, the dashboard is composed of aggregated information and does not allow for the identification of individual patients.

Based on the discussion outlined above, it can be concluded that the proposed dashboard fulfills the formulated requirements. However, additional evaluation efforts can increase the support for the dashboard. The dashboard might for instance be used in practice, after which the users can be queried on their experiences with respect to the requirements.

\section{CONCLUSION}

This paper presents a dashboard providing real-time information on ED crowding. Besides general real-time patient and patient flow statistics, a series of crowding indicators are provided. To maintain the dashboard's clarity, the most desirable indicators are selected using a two-round multi-centric Delphi study. The provided information will enable staff members to proactively judge whether corrective actions are required. The dashboard is developed using the principles of design science research, which provides a solid methodological underpinning for the research process.

Besides the aforementioned contributions, two limitations of the study also need to be recognised. Firstly, the evaluation of the dashboard involves studying the degree in which it satisfies the specified requirements. Additional support for the importance of the proposed dashboard would originate from studying its use on the working floor. This would highlight the dashboard's contribution to ED practice. Secondly, only Flemish EDs and experts are involved in the two-round modified Delphi study. Hence, other crowding indicators might be judged desirable when an international panel is consulted.

Future work can improve the information value of the current dashboard by, e.g., including color schemes which reflect positive or negative evolutions in an indicator's value. Moreover, 
This is an Accepted Manuscript of an article published by Elsevier in International Emergency Nursing, available online: https://doi.org/10.1016/j.ienj.2017.08.001

further evaluative efforts can involve studying the use of the dashboard on the working floor. While the prior recommendations are situated within the scope of the current dashboard, the functionality scope can also be extended. In this respect, integrating prediction and real-time simulation in the dashboard are promising directions. This can enable the dashboard to provide recommendations on potential courses of action to improve patient flow when the ED is crowded.

\section{APPENDIX - EMERGENCY DEPARTMENT DASHBOARD}

In this appendix, an overview of the seven content tabs of the developed dashboard is presented.

\section{Overview tab}

The overview tab, which is opened in Figure 3, is composed of three graphs which present an overview of the current situation at the ED. The upper graph shows the evolution of patient in- and outflow on the current day with a one-hour interval. While patient inflow is visualized by a stacked bar chart with triage code as a grouping variable, patient outflow is represented using a line. The two graphs at the bottom provide insight in the composition of the patient population by representing their age and the ED unit to which the patient is assigned. As is the case for the in- and outflow graph, colors reflect the composition of a patient group in terms of triage code.

\section{Time interval tab}

The first round of the modified Delphi study shows that not all time intervals defined by Wiler et al. (2015) are judged equally desirable. Consequently, five time intervals, all scoring above 5.5 on a 7-point desirability Likert scale on average, are selected for inclusion in the time interval tab. These are the length of stay, boarding time, the time until the allocation of a hospital bed, the radiology interval and the laboratory interval. As such time intervals tend to have a positive skew, both the mean and median are included in the dashboard. Compared to the mean, the median is influenced less severely by outliers. Besides 
This is an Accepted Manuscript of an article published by Elsevier in International Emergency Nursing, available online: https://doi.org/10.1016/j.ienj.2017.08.001

presenting the mean and median values for the current situation, the dashboard also depicts the evolution in the time interval values. By clicking on one of the intervals, one-hour boxplots are shown for each of the past six hours. This is illustrated in Figure A1, where length of stay is selected.

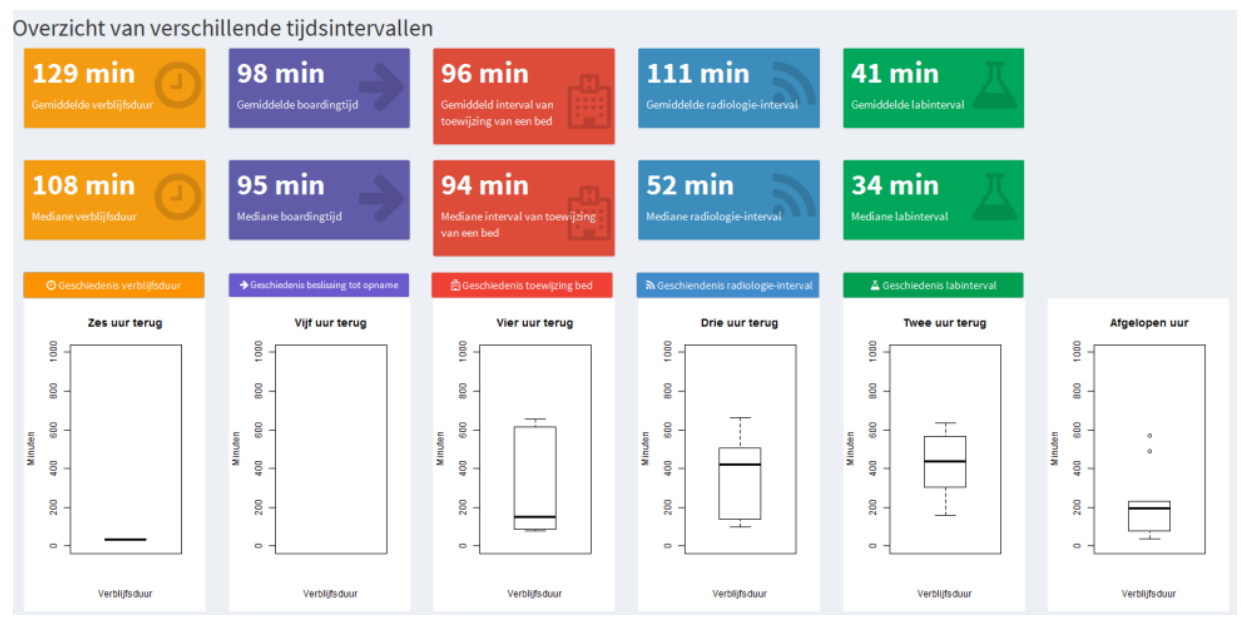

Figure A1: Screenshot of the time interval tab

\section{Input - throughput - output tab}

The input-throughput-output tab, illustrated in Figure A2, contains eight measures formulated by Solberg et al. (2003). The patient volume standardized by the number of bed hours, the most frequently occurring triage code and the number of ambulance patients per bed hour are included as input measures. The two throughput measures are the median length of stay and the workload per bed hour. Three output measures are included: the median boarding time, the boarding pressure and the average discharge time interval. All the selected measures have an average score above 5.5 on a 7-point Likert scale of desirability during the first round of the modified Delphi study. For the patient volume per bed hour, the median length of stay and median boarding time, graphs are included depicting the evolution of these measures' values during the past eight hours. This can be seen at the bottom of Figure A2. 
This is an Accepted Manuscript of an article published by Elsevier in International Emergency Nursing, available online: https://doi.org/10.1016/j.ienj.2017.08.001

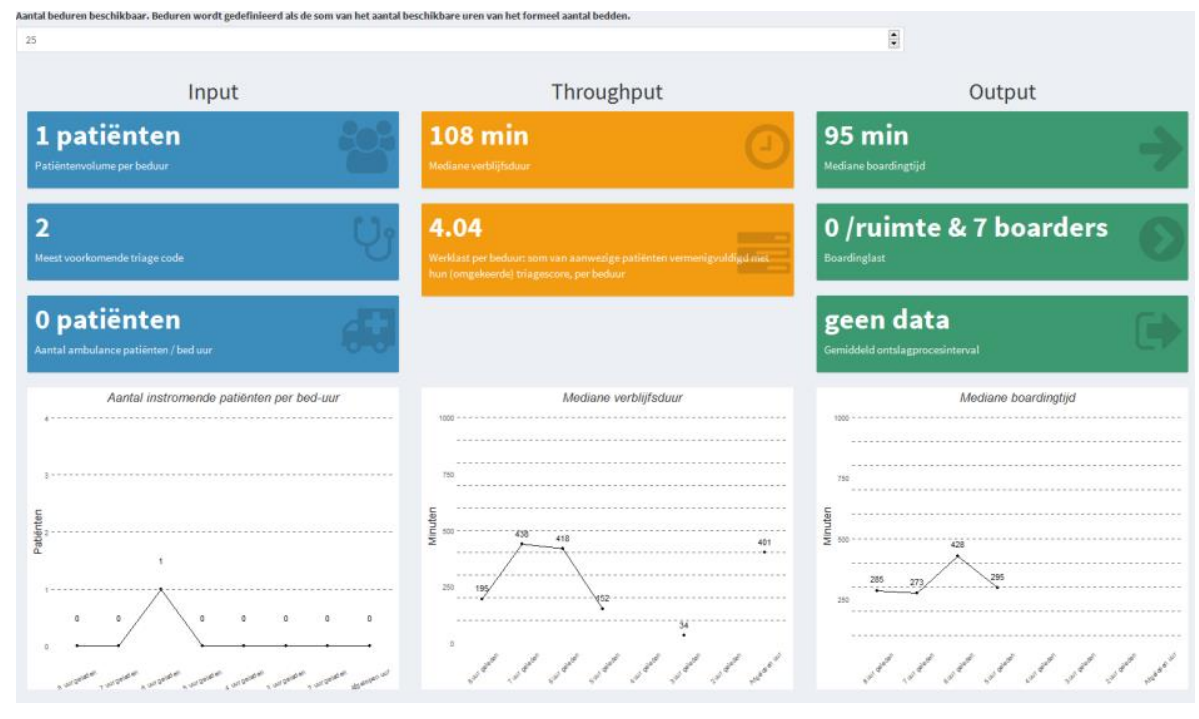

Figure A2: Screenshot of the input-throughput-output tab

\section{READI tab}

Of all quantitative crowding measurement scales, the Real-time Emergency Analysis of Demand Indicators (READI) achieved the highest desirability score in the first round of the modified Delphi study. The READI score is calculated based on three crowding indicators, i.e. the bed ratio, the acuity ratio and the provider ratio (Hoot et al., 2007). Even though an expert expressed doubts regarding the use of READI as a predictor of crowding in the second round, it is still included in the dashboard given the strong support during the first round. As shown in Figure A3, the READI tab contains both the READI score and its three building blocks.

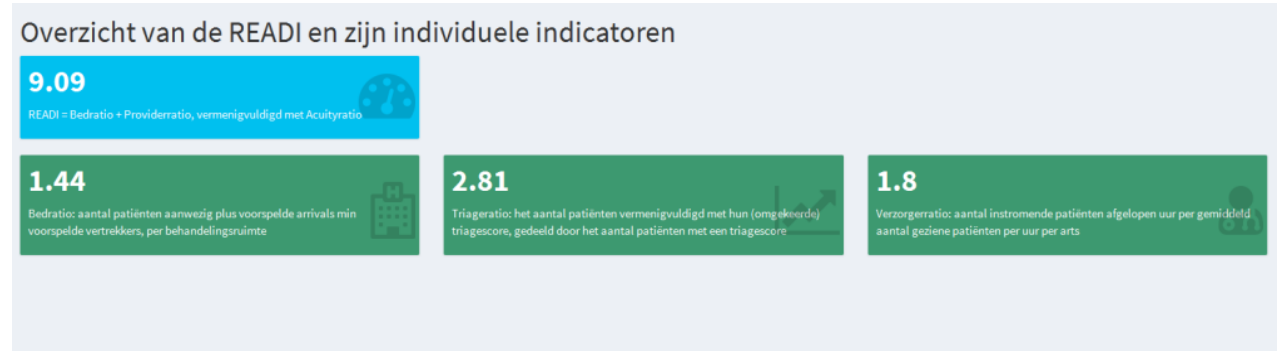

Figure A3: Screenshot of the READI tab 
This is an Accepted Manuscript of an article published by Elsevier in International Emergency Nursing, available online: https://doi.org/10.1016/j.ienj.2017.08.001

\section{ED Work Score tab}

The ED Work Score (Epstein and Tian, 2006) also received a high desirability score in the modified Delphi study. The layout of the corresponding dashboard tab is similar to the structure of the READI tab as it also depicts the aggregated ED Work Score and its individual indicators. These individual indicators are the number of patients waiting per bed, the acuity per nurse and the number of boarding patients per bed. A screenshot of the ED Work Score tab is provided in Figure A4.

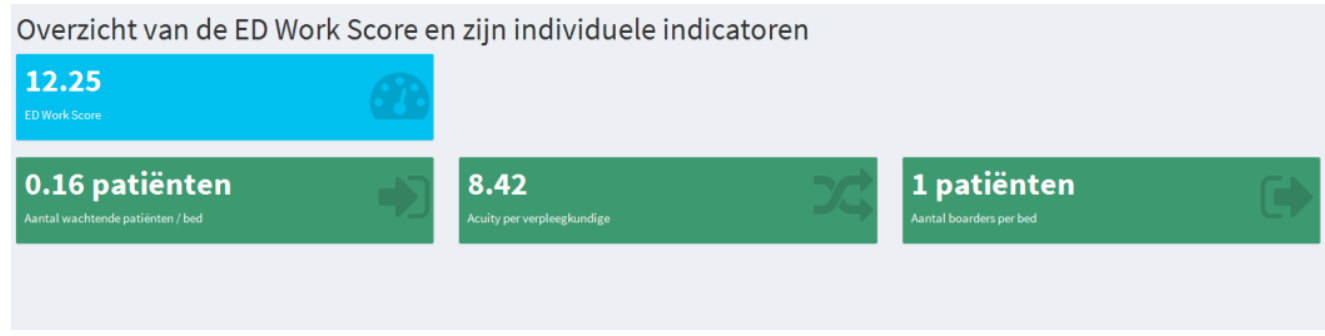

Figure A4: Screenshot of the ED Work Score tab

\section{ICMED tab}

Based on the results of the modified Delphi study, ICMED (Boyle et al., 2013) should not be included in the dashboard with a mean desirability score of 5 . This might be attributed to the fact that it is proposed quite recently. As ICMED refrains from making a binary judgment on whether or not the ED is crowded, it is included in the dashboard. As shown in Figure A5, the ICMED tab includes the indicators in the middle and the number of threshold transgressions at the top. At the bottom of the tab, the number of boarders for which a hospital bed is available and the number of boarders that is waiting for a bed for over two hours are shown. These values are added as they can be more informative than the associated ICMED indicators which are expressed as percentages. 
This is an Accepted Manuscript of an article published by Elsevier in International Emergency Nursing, available online: https://doi.org/10.1016/j.ienj.2017.08.001

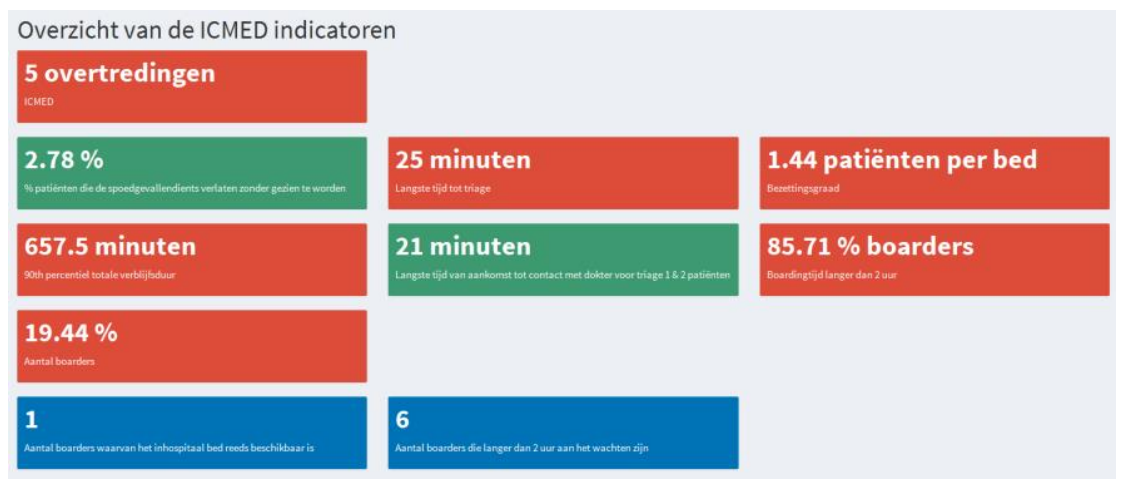

Figure A5: Screenshot of the ICMED tab

\section{Quality and safety indicator tab}

The analysis of ED's operations currently focuses on quantifying the degree of crowding. Finding appropriate measures for this purpose has proven to be a challenging task. Quality of care and patient safety can also be influenced by crowding. However, quality and safety indicators still need to be developed, taking into account efforts such as the Workload Indicators of Staffing Need developed by the WHO (WHO, 2017). Moreover, the influence of ED occupancy on these indicators needs to be measured to determine the required ED capacity and, consequently, a threshold for crowding (Bergs et al., 2016). The critical importance of quality of care and patient safety requires their inclusion in a dashboard that aims to provide comprehensive support to ED staff. Following this line of reasoning, a quality and safety indicator tab is already added in the dashboard's conceptual overview in Figure 2. 
This is an Accepted Manuscript of an article published by Elsevier in International Emergency Nursing, available online: https://doi.org/10.1016/j.ienj.2017.08.001

\section{REFERENCES}

Bergs, J., Vandijck, D., Hoogmartens, O., Heerinckx, P., Van Sassenbroeck, D., Depaire, B., Marneffe, W., Verelst, S. Emergency department crowding: time to shift the paradigm from predicting and controlling to analysing and managing. International Emergency Nursing. $2016 ; 24: 74-7$.

Boyle A, Beniuk K, Higginson I, Atkinson P. Emergency department crowding: time for interventions and policy evaluations. Emergency Medicine International. 2012;2012(2):1-8.

Boyle, A., Coleman, J., Sultan, Y., Dhakshinamoorthy, V., O'Keeffe, J., Raut, P., Beniuk, K. Initial validation of the International Crowding Measure in Emergency Departments (ICMED) to measure emergency department crowding. Emergency Medicine Journal. 2013; 32:105-8.

Dresch, A., Lacerda, D.P., Antunes, J.A.V. Design science research. Heidelberg: Springer, 2015.

Epstein, S.K., Tian, L. Development of an emergency department work score to predict ambulance diversion. Academic Emergency Medicine. 2006; 13(4):421-6.

Hasson F, Keeney S, McKenna H. Research guidelines for the Delphi survey technique. Journal of Advanced Nursing. 2000; 32(4):1008-15.

Hoot, N.R., Zhou, C., Jones, I., Aronsky, D.. Measuring and forecasting emergency department crowding in real time. Annals of Emergency Medicine. 2007; 49(6):747-755.

Johannesson, P., Perjons, E. An introduction to design science. Heidelberg: Springer, 2014.

Keeney S, Hasson F, McKenna HP. A critical review of the Delphi technique as a research methodology for nursing. International Journal of Nursing Studies. 2001; 38(2):195-200. 
This is an Accepted Manuscript of an article published by Elsevier in International Emergency Nursing, available online: https://doi.org/10.1016/j.ienj.2017.08.001

Niehaves, B. On epistemological diversity in design science: new vistas for a design-oriented IS research?. Proceedings of the 2007 International Conference on Information Systems. 2007.

Peffers, K., Tuunanen, T., Rothenberger, M. A., Chatterjee, S. A design science research methodology for information systems research. Journal of Management Information Systems. 2007; 24(3): 45-77.

Pines J.M., Hilton J.A., Weber E.J., Alkemade A.J., Shabanah Al. H., Anderson P.D., et al. International perspectives on emergency department crowding. Academic Emergency Medicine. 2011; 18(12):1358-70.

Pitts SR, Pines JM, Handrigan MT, Kellermann AL. National trends in emergency department occupancy, 2001 to 2008: effect of inpatient admissions versus emergency department practice intensity. Annals of Emergency Medicine. 2012; 60(6):679-686.e3.

R. Available at: https://www.r-project.org [Accessed 8 Sep. 2016].

Shiny. Available at: http://shiny.rstudio.com [Accessed 8 Sep. 2016].

Shinydashboard. Available at: https://cran.r-project.org/web/packages/shinydashboard [Accessed 8 Sep. 2016].

Solberg, L.I., Asplin, R., Weinick, R.M., Magid, D.J. Emergency department crowding: consensus development of potential measures. Annals of Emergency Medicine. 2003; 42(6):824-834.

Tufte, E.R. The visual display of quantitative information. Graphics Press: Cheshire, 2001. 
This is an Accepted Manuscript of an article published by Elsevier in International Emergency Nursing, available online: https://doi.org/10.1016/j.ienj.2017.08.001

Verelst S, Wouters P, Gillet J-B, Van den Berghe G. Emergency department crowding in relation to in-hospital adverse medical events: a large prospective observational cohort study. The Journal of Emergency Medicine. 2015; 49(6): 949-61.

Wieringa, R. Design science methodology. Heidelberg: Springer, 2014.

Wiler, J.L., Welch, S., Pines, J., Schuur, J., Jouriles, N., Stone-Griffith, S. Emergency department performance measures updates: proceedings of the 2014 emergency department benchmarking alliance concensus summit. Academic Emergency Medicine. 2015; 22(5):542-553.

WHO, Workload indicators of staffing need (WISN). Available at: http://www.who.int/hrh/resources/wisn_user_manual/en/ [Accessed 21 June 2017]. 